LCRL-52957 Rev. I

Distribution Category

UC- 21,32

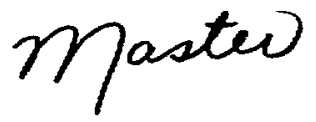

\title{
An introduction to Praxis
}

\author{
James R. Greenwood \\ Arthur Evans, Jr.* \\ C. Robert Morgan* \\ Michael C. Zarnstorff ${ }^{\dagger}$
}

Manuscript date: December 3, 1980

*Bolt, Beranek, and Newman, Inc.

Cambridge, MA

\footnotetext{
${ }^{\dagger}$ University of Wisconsin
}

Physics Department

Madison, WI

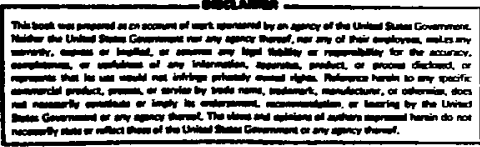




\section{CONTENTS}

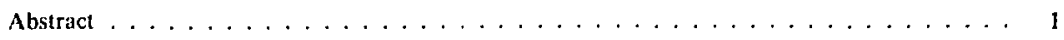

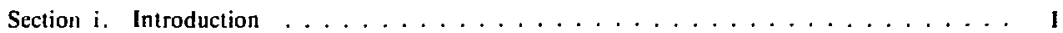

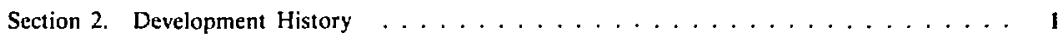

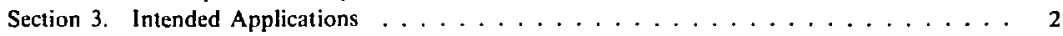

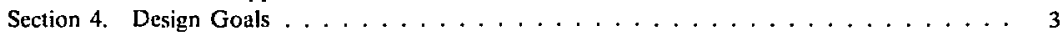

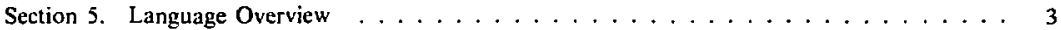

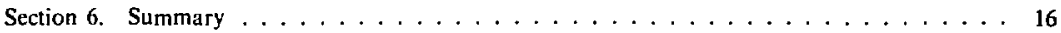

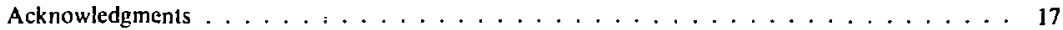

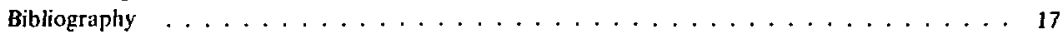

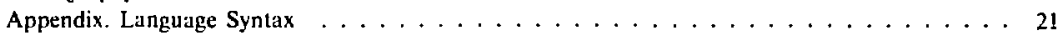




\title{
An introduction to Praxis
}

\begin{abstract}
Praxis is the practice of the programming art, science, and skill. It is a high-order language designed for the efficient programming of control and systems applications. It is a comprehensive, strongly typed, block-structured language in the tradition of Pascal, with much of the power of the Mesa and Ada languages. It supports the development of systems composed of separately compiled modules, user-defined data types, exception handling, detailed control mechanisms, and encapsulated data and routines. Direct access to machine facilities, efficient bit manjpulation, and interlocked critical regions are provided within Praxis.
\end{abstract}

Keywords: Praxis, high-level control language, compilers, real time.

\section{Section 1 \\ INTRODUCTION}

This report describes the control-system implementation language Praxis, which has been developed in the Laser Fusion Program at the Lawrence Livermore National Laboratory (LLNL) for control applications. It serves as an introduction to the language so that the reader can get a feel for what the language is and find out if it is applicable to the reader's needs.

Most of the report consists of graduated examples that provide an overview of the language. The definition and details of the language can be found in the Praxis: Language Reference Manual and in other companion reports that follow the publication of this report.

\section{Section 2}

\section{DEVELOPMENT HISTORY}

In the summer of 1978 , it became apparent in the laser fusion program at LLNL that we needed a control-oriented language for use in programming the control system of the Nova laser system. Our experience in developing the laser control system for Shiva, consisting of 55 processors, clearly indicated that if a controls-oriented programming language were available we could save considerable time and effort with respect to Nova.

After carefully evaluating potential languages, including DOD's current development of Ada, we chose to implement Praxis. Although Ada would meet our needs, it would not be available in time for Nova (compilers had to be available before the mid-1980's to meet the needs of the Nova controls programming). In retrospect, our selection of Praxis proved correct. since a Praxis compiler now exists and is in use while the more ambitious Ada development is still ongoing. 
The development of Praxis originated from an initial study by Bolt, Beranek, and New man (CBN), Inc., funded by the Defense Communications Agency (DCA.), to determine the requirements of a language for communications programming. The result of that study (BBN Report 3261) concluded that no current language fulfilled the rigorous needs of communications programming.

The DCA then funded BBN to design an appropriate programming language. This resulted in a preliminary design of the COL language described in BBN Report 3534, May 1977 (A. Evans, C. R. Morgan). Also, the DCA funded BBN to design a compiler described in BBN Report 3533, May 1977.

In January 1979 LLNL funded $B \bar{B} N$ to augment the design of COL and to implement a COL compiler for the PDP- $i$ i series of computers from Digital Equipment Corporation. With the clarification of the Nova controls design and schedule, BBN's work has been expanded to include the development of a VAX/VMS native-mode compiler, documentation, additional language design, and a high-level input/output package. BBN is scheduled to complete their work by fall 1980, with the delivery of documented operational compilers for Praxis, on both the PDP-11/RSX-11 and VAX/VMS systems, written in Praxis.

In January 1980 we changed the name of the language from COL to the current Praxis. We felt that the language had evolved significantly from that of the original COL study and that a new name would better reflect its power.

In March 1980 the preliminary PDP- 11 compiler successfully passed two critical milestones. The first milestone was that the compiler, which is written in Praxis, had to compile itself successfully on the PDP-11/RSX-1IM system. This would demonstrate that the compiler was self-supporting on the PDP-11 systems, and that the bulk of compiler was correctly implemented.

The second milestone was the implementation of a Nova controls application of the language, for a RUM-based LSI-1I processor. A 2000-line assembly-language, steppermotor control program had to be recoded in Praxis, compiled, and burnt into read-only memory (ROM). This would demonstrate that the language was indeed powerful enough to replace detailed, assembly language sequences and that the compiler correctly implemented the controls-oriented features.

\section{Section 3}

\section{INTENDED APPLICATIONS}

Praxis is designed for programming control and communication applications. It is also useful for system programming applications, which require many of the same language facilities found in Praxis. All these applications impose stringent requirements on programming in such areas as

- Efficiency of object code.

- Direct access to machine facilities.

- Efficient bit manipulation.

- Complex data and control structures.

- Large programs developed by a team.

- Maintenance and upgrades.

The programming of these applications requires detailed control of the compiler-produced code, the optimization, the variable allocation, and the run-time support. In these applications, it is important for the programmers to explicitly control exactly what is going on. 


\section{Section 4}

\section{DESIGN GOALS}

The design goals of Praxis are based on the requirement of the language being a useful tool for programming control applications. Consequently, the goals may be stated as follows:

- Efficiency: first of the compiled code, then of the compiler.

- Readability: particularly more important than writability.

- Completeness; in the sense that

* it must be possible to program all of any one application in Praxis without recourse to assembly language.

* it must be possible to write the compiler for Praxis in the Praxis language.

- Portability: Praxis should be reasonably machine-independent.

- Modularity: it must be possible to program large projects within Praxis, requiring separate compilation of modules and configuration control.

- Usability: primarily used by experienced programmers, so that the ease of learning Praxis is less important than the ease of using Praxis.

The primary requirements for control applications are efficiency of the compiled code, completeness. and portability. Praxis must produce programs that make effective use of hardware resources directly controlled by the programmer. Also, the programs should bc as portable as possible between machines. In general, the language features are portable but. where machine-dependent parts are necessary, they are as conspicuous as possible. For example, the programmer can override the language's type-checining mechanism, but it is easy to see when this is being done.

The requirement for efficiency has had one other impact on the language design. All proposed features and facilities have to be scrutinized for the run-time and the compile-time efficiency of their implementation. No matter how desirable a particular feature might be, it had to be rejected if a reasonably efficient implementation could not be designed.

\section{Section 5}

\section{LANGUAGE OVERVIEW}

Praxis is a modern, block-structured, fully typed, algorithmic progi umming language in the tradition of Pascal. Its design has been influenced by the languages Simula, BCPL, Euclid, PL/I, Jovial, CS-4, Alphard, Mesa, and Bliss languages, as well is by the DOD's language development work and the proposed Ada language. In scope and power, Praxis most closely resembles Ada and Mesa.

Since the control environment differs in important ways from application to application and machine to machine, Praxis has features to handle these differences. High-level facilities that mask machine dependencies and foster machine independence (portability) usually prevent the use of exactiy the programming capability needed for real-time. control applications programming. However. Praxis is a high-level language that has controlled access to machine dependencies.

Praxis is strongly typed. The programmer is given a collection of predefined types and has the ability to construct new types. Every variable. constant, parameter. and expression has a type. All types tan be deduced at compile-time and the compiler requires that each value be used in a way that is consistent with the rules associaled with its type. For instance. 
it is a compile-time error to attempt to pass an integer parameter to a routine that iequires a real parameter.

The language is blocked structured. Blocks are a method of packuging statements and declarations so that the scope of the statements is clearly specified and controlled. Praxis has more than 10 block-structured statements, each of which is delimited by an $X X X /$ end $X X X$ pair, where $X X X$ represents the particular statement name. For instance:

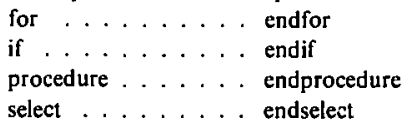

The block structuring also enforces a particular programming style that is more readable and maintainable than that of unstructured programming.

A simple example in the language is the matrix multiply of two $N$ by $N$ matrices named SpecA and SpecB and storing the result in Spectrum:

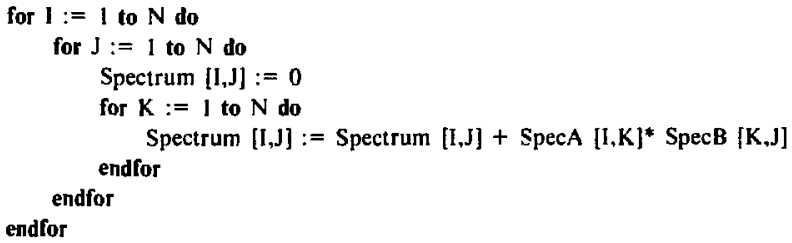

This example only makes sense within the scope of the declarations for the variables used. All the variables, except the one for loop indices, must be declared before use. Thus, the code above would be preceded by something of the form

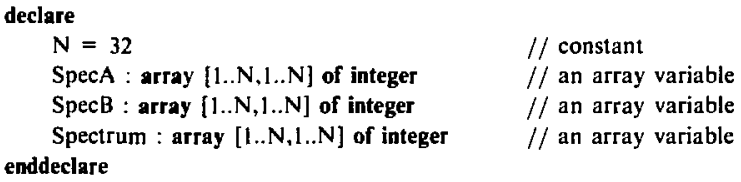

This declaration block could be written more concisely in various forms. One method would be to use a user-defined type for the array declarations. which then would ensure that the three arrays are all the same type and remain so with subsequent software maintenance. Thus, the declarations could take the form

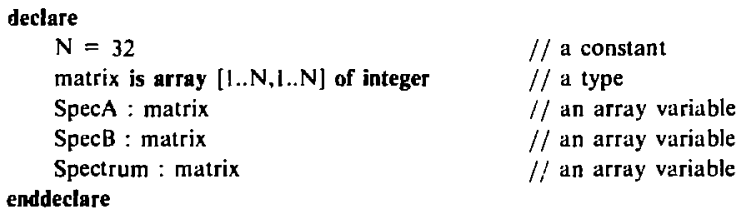

Note that we have used the language's comment convention " $/ /$, " which designates that all text to the right on the line is treated as a comment. Here, all language-reserved words are boldface in the examples, but no distinction is made in actual programs. 
Another example is a simple exchange sort in which a values array is sorted into ascending order:

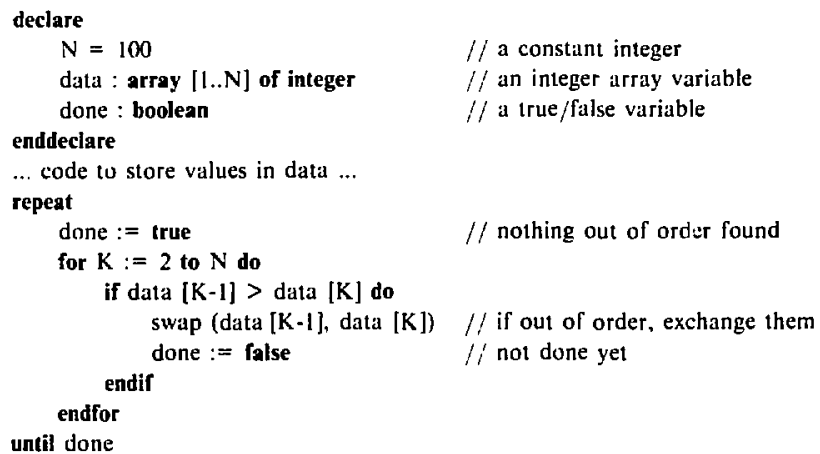

The repeat block-structured statement is the exception to the ending syntax rule. in that the until is the end for the repeat block. The repeat/until has the semantics that the included statenents are executed repeatedly until the expression after the until is true. Other looping constructs are available in Praxis, including the while/endwhile, and four forms of for/endfor.

A more detailed control programming application is shown below. It directly reads a hardware input/output device on a PDP-11 computer in a multi-process environment. In this example. the resource (i.e., $1 / O$ device) is protected by the interlock variable padlock in a critical region. Another process with similar code. using the same resource. cannot preempt the critical-region code sequence.

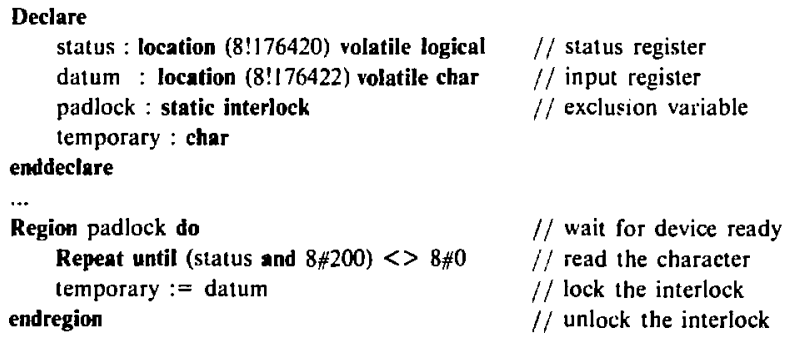

The attribute volatile on the variables status and darum informs the compiler that the variables must be referenced directly each time they are mentioned in the program, and no optimizations are to be performed on these variables. It allows variables to be used as $1 / 0$ registers, as above, as well as to be used in shared memory.

The location attribute informs the compiler to place the variable in the physical address specified by the octal (8!) integer constant in the purentheses. The variable is static and always resides at that locution. The static interlock is at a fixed location determined by the compiler.

The logical predefined data type may be thought of as a bit-string data type on which bit-by-bit operations may be performed. In the until clause, a bit in the status variable is teited by the bit-by-bit and with the octal ( $8 \#)$ logical constant and comparison to a lugical itro. 
$\Lambda$ more complex application, which demonstrates the ability in Praxis to bypass the strong typing (when desired), is the sequence that extracts the cxponent value from a real number on the PDP-11:

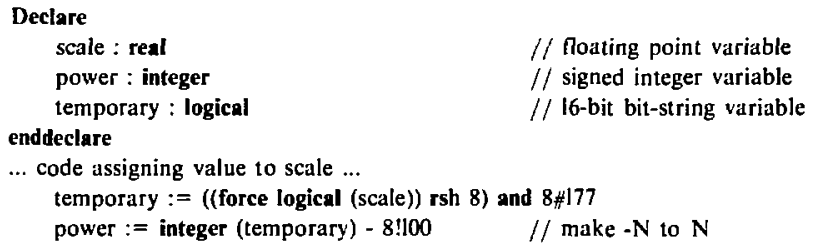

The force explicitly overrides the type-checking mechanism and specifies that the variable scale is to be handled as a logical in this expression. The logical value (i.e., 16 bits) is shifted right 8 bits and masked with the logical constant. Temporary is assigned the resulting value that was the exponent of the real variable scale. The logical value is then converted to an integer and stored in the variable power.

Note the distinction between force and type conversion; force informs the compiler to treat a variable as a particular type regardless of its actual type: conversion causes the variable to be converted to the desired type.

Another application of type conversion is shown in the function upper, which converts a possible lower-case letter to an upper-case letter:

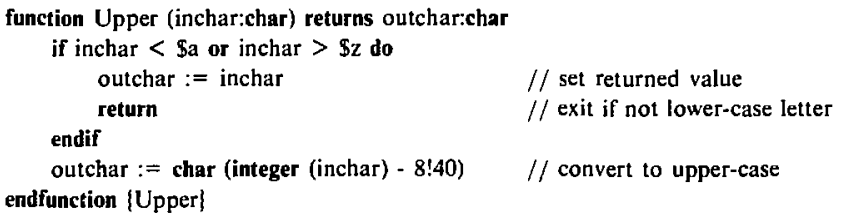

The previous function example utilized the return statement for explicit exit from a routine (i.e., procedure or function). This statement is one of several such statements that eliminates the need for a GOTO in the language. An important feature in the language is the lack of the GOTO statement. The following example uses two other control flow statements, together with block labeling, to program an application that normally requires a GOTO statement.

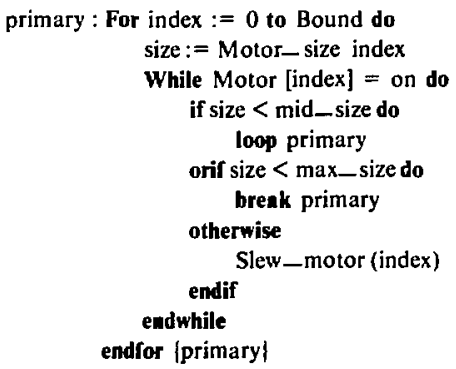

// labeled statement

$/ /$ assignment

$/ /$ inner loop

$/ /$ conditional statement

$/ /$ iterate for loop

$/ /$ alternative

// exit for loop

// default alternative

$/ /$ procedure invocation

$/ /$ end of alternatives

$/ /$ end of inner loop

$/ /$ end of labeled block 
The loop statenient above causes the for loop iteration to occur: that is, it acts like a GOTO the for. which causes the iteration count of the loop to be incremented, the test for completion to be performed, and the for block to be executed if the iterations have not been completed. The break statement on the other hand is a block exi: statement. In the above case. it exits three levels of blocks: the if, while, and for, and exerution continues after the endfor. Labels can only appear on blocks (at the beginning and end) and are only used with the break, loop, and retry (in critical regions) statements.

The statement sequence above would have had to be preceded by a declaration in which the variables, types, and constants are declared. All items must be declared before their use. The declaration for the above could be

Declare

$$
\begin{aligned}
& \text { min-size }=0 \\
& \text { mid_size }=25 \\
& \text { max_size }=50 \\
& \text { size : integer initially min size } \\
& \text { bound : integer initially } 0 \\
& \text { Onoff is [on, off] initially off } \\
& \text { Motor_size: array [0..9] of integer } \\
& \text { Motor : array [0..9] of Onoff }
\end{aligned}
$$$$
\text { enddeclare }
$$

$$
\begin{aligned}
& \text { // a constant } \\
& / / \text { a variable } \\
& / / \text { an enumeration type } \\
& / / \text { array variable } \\
& / / \text { array variable }
\end{aligned}
$$

Notice the use of initialization clauses on variable and type declarations, which allow for variables to be declared with initial values. For instance, the variable bound is declared with the initial value zero, and the variable motor is declared as an array of enumerated data values, initially all elements being the value off. The declaration form is declares new data types and is discussed more fully below.

The break and loop example above also introduced the multiarm if statement that allows the programming of a branch-tree. Only one arm of the statement is elaborated on each iteration of the while loop, depending on the boolean expressions in each arm. Any number of orif clauses may be present, and the otherwise clause is optional. Thus, the forms below are valid if statements:

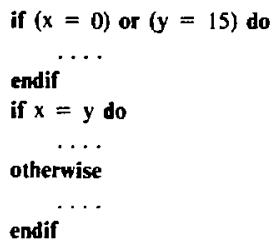

Another form of flow control statement in Praxis is the select statement, which selects a sequence to elaborate from a set of cases according to a selection expression. For example:

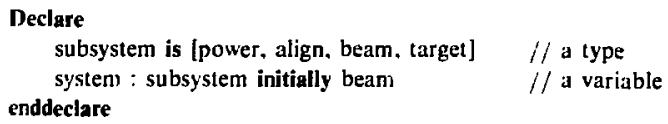




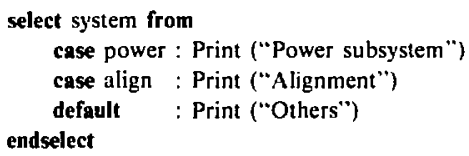

Only one of the Print procedure invocations is executed, depending on the value of the enumerated variable system. Note that the default clause will be executed for any values other than power or align. The strong typing and declarations ensure that the only other enumerated values the system can take on are beam and target.

Another control application that can be run on the PDP-11 uses data structures, procedure variables, and interrupt procedures to quickly and easily program an application that normuliy must be done in assembly language:

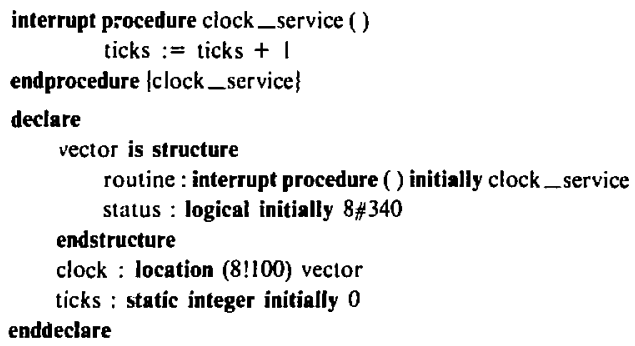

The variable ticks gets incremented for each interrupt from the line clock on the PDP-11.

Note that because the interrupt procedure is executed asynchronously, communication with the other code must be done through static variables. Only one copy exists of any static variable.

The user-defined structure data-type vector has two fields: the first is the routine. which is of type Interrupt Procedure and is initialized to be the address of the clock service routine; the second field is a logical (i.e., bit-string) variable, which is set to the value desired for the processor status word. The actual declaration and positioning of the clock vector are accomplished by the variable declaration clock and the location attribute.

The above sequence would most likely be used in conjunction with a read routine of the form

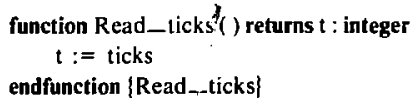

The empty parentheses (i.e., (1) denote a routine with no parameters and would be invoked with the form

$$
\text { count := Read__ticks ( ) // get \# of ticks }
$$

The interrupt-procedure example utilized the structure data type (i.e., the userdefined vector) and the procedure data type. These data types are two of the predefined dati types in the language, all of which are listed below: 


\section{Diserete types}

$$
\begin{array}{ll}
\text { integer } & - \text { signed } \\
\text { cardinal } & - \text { unsigned integer } \\
\text { char } & - \text { ASCII character } \\
\text { boolean } & - \text { true/false } \\
\text { enumeration } & - \text { programmer-specified values }
\end{array}
$$

Control types

$$
\begin{array}{ll}
\text { interlock } & \text { - locked/unlocked } \\
\text { logical } & \text { - bit string } \\
\text { pointer } & \text { - pointer } 10 \text { a typed object }
\end{array}
$$

Floating types

real

- floating point

long real

- double precision real

Aggregate types

array

st ructure

set

Routine types

procedure

function

(Other types

general descriptor
- array of any type. access by index

- various type components. access by name

- set of discrete type

- typed procedure variables

- typed function sariables

- anion of all types (used as formal parameter)

- lype descriplor

Liser-delined data types may be characterized in terms of the predelined types or other user-defined types. The is form declares a user-delined dat type. The semaphore in the example below is a user-defined data type. Sync is a variable of type semaphore:

\section{declare}

semuphore is structure

$\therefore$ Iype decl

$$
\text { lock : interlock }
$$

count : integer initially 0

endstrueture

Sync : semaphore

it semaphore variable

\section{enddeclare}

This method for synchronization was proposed by Dijkstra in 1968. The semaphore is a special variable that can be maripulated only by the primitives Wait (also called the $P$ operator) and Signal (also called the $V$ operator). defined as follows:

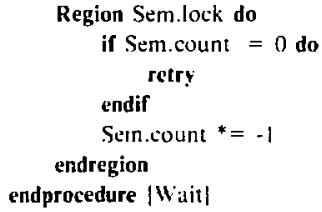

$\therefore$ P operator protect count access check count value loop. unlock. a relocin lock.

decrement count end of critical region return from procedure

i/ V operator enter crilical region 


$$
\begin{aligned}
& \text { Sem.count } *=+1 \quad / / \text { increment count } \\
& \text { endregion } \quad / \text { ! exit critical region } \\
& \text { endprocedure JSignaly } \quad / / \text { exit from Signal }
\end{aligned}
$$

The Wait procedure allows a process to delay while waiting for an event to occur. The Signal procedure is used to signal another process that an event his occurred. In the above example. it is assumed that the semaphore would be shared between two processes. and each process would have its own copy of the Wair and Signal procedures. The interlock is utilized to guarantee atomic access to the semaphore count without worrying about actual code sequences.

The form "* $="$ assignment statement can be read as transformed by. Thus, the statement

$$
\text { Sem.count } *+1
$$

increments the coumt field of the semaphore passed as an argument to Signal and is equivalent to the statement

Sem.count $:=$ Sem.count +1

The formal parameter specification on Wait (and Signal) explicitly specifies that the actual parameter be passed by Ref (i.c., reference) and that the parameter will be both read (i.t.. in) and written (i.e., out). Parameters may be pissed by Ref or Val (i.e., value. by copy) with the default being by Val. The programmer would usually specify by Ref, for large aggegates, in the interest of efficiency. The dita-passing direction can be specified as in, inout, or out with the default being in. The compiler checks at compile-time to cnsure that the usage of the parameter, within the routine, is consistent with the passing direction.

The semaphore, Wait, and Signal definitions can be encapsulated within a Module for separate compilation, or for data abstraction, or for both. Thus, the definition module would be

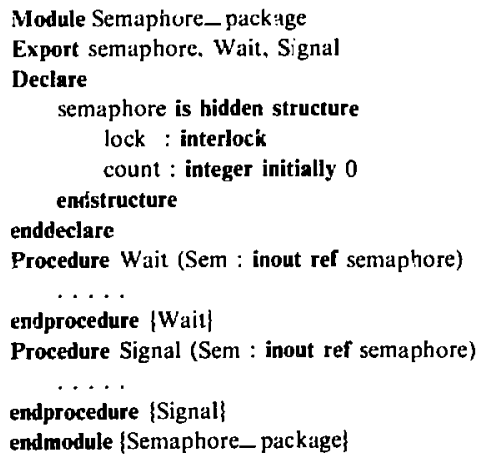

The declarations of semaphore, Wait, and Signal are made available by the Export to other modules (i.e., if this module wis within another) or to other separately compiled modules that Import the declarations. Note that typ a well as data and routines, cin be imported and exported. 
The hidden altribute specitied on this new declaration of the semaphore type implements what is referred to as an abstract data type. That is, the type name is known outside of the module. but the internal structure is unknown. Thus, an application program can itnport the type and declare and use variables of type semaphore without knowing the details of the structure. For instance:

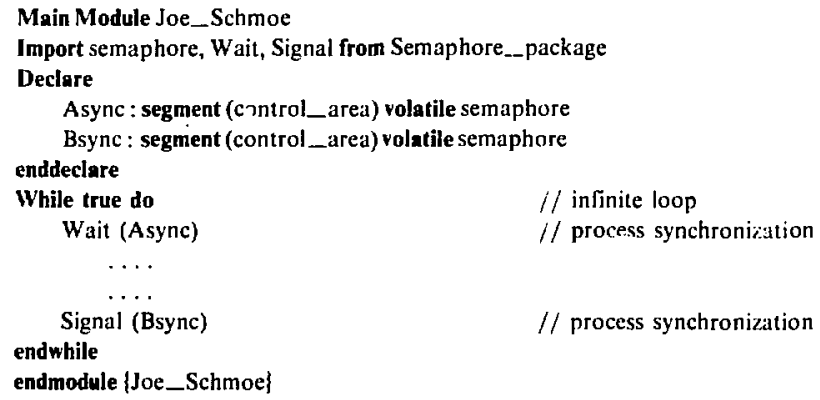

The main module allows the use of top-level code (i.e., code not within a routine) and is the main program or process, depending on the operating system employed. In the example, 1wo variables. Async and Bsync, are declared, using the imported semaphore definition. These variables are then used with the Wait and Signal procedure calls to synchronize this process with other processes. Note that the language makes no assumptions about the run-time system; no tasking or multiprocess operations are built into Praxis. These facilities can be programmed in the language, or provided by existing operating environments.

The segment storage class on the declarations of $A s y n c$ and Bsync specify that the semaphores are slatic in a named (i.e., control area) data area. This data area can be associated with program sections or location counters (depending on the implementation) by means of the \%Segment compiler directive. For instance, for a PDP-11/RSX-11M implementation, the directive

\%Segment control_area $=\mathrm{RW}, \mathrm{D}$

creates a program section (i.e., PSECT) which can be controlled and positioned at link-time. Segment can be viewed as a named location.

The Print routine used in a previous example could be written as

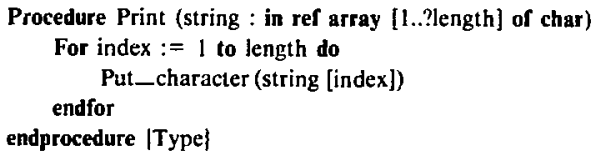

The formal parameter specilies a flexible array of characters as the type of the parameter: this allows the arrays of characters of any length to be passed, with an implicit-size parameter length. A quoted string is considered an array of characters indexed 1 througil $N$, where $N$ is the number of characters between the quotes.

Flexible arrays can also be allocited from the free memory storage (i.e.. heap) and aecessed through pointers. The free memory is only utilized when the programmer explicitly 
specifies it by the allocate and free operations. There is no implicit heap usage or garhage collection in the language, an essential requirement in real-time control applications. Data objects in the heap are referenced by pointers. For instance:

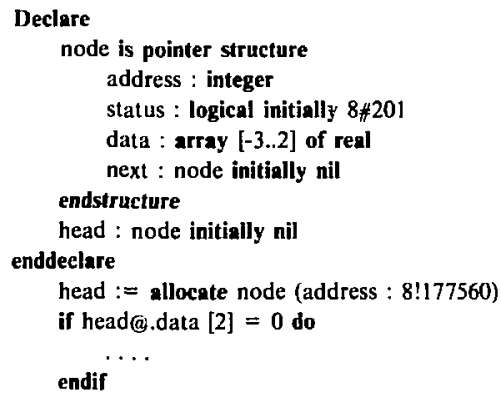

The node declaration is a pointer to a structure of the form shown. Head is a declaration of a pointer object, and the assignment statement creates an object within the heap and places the location of the object in the variable head. The field address will be initialized to the octal value 177560 , and the field status will be initialized to the octal value 201 via the type initialization clause.

The object is referred to with the "(a)" operator. That is, since head is a pointer to a structure, then

$\begin{array}{ll}\text { head@ } & - \text { whole structure } \\ \text { head@.address } & - \text { an integer field } \\ \text { head@.data [J] } & - \text { an element of a field } \\ \text { head@.next } & - \text { a field } \\ \text { head@.next@.address } & - \text { a field of an object pointed to by a field }\end{array}$

are valid references. Note that the last reference only makes sense if the value in the next field points to something (i.e., not nil).

The node pointer structure allows a linked list to be allocated at runtime from the heap. The iterator form of the for loop is useful for stepping through such a list.

For $p:=$ head then $p @$.next while $p<>$ nil do

if p@.status and $8 \$ 200<>8 \# 0$ do

endif

endfor

The pointer variable $p$ is $d t$. lared and is assigned the value from head; if the value is not nil then the body of the for block is elaborated. The expression between the then and while is the iteration expression that specifies the subsequent values of $p$.

Objects allocated from the heap must be explicitly returned with the free procedure, which has the form 
Free may be called with any type of pointer and any number of parameters.

An important consideration in real-time systems is the ability to handle abnormal conditions and catastrophic failures. In Praxis, this is accomplished with named exceptions and guard blocks. Both predefined and user-defined exceptions are available and can be caught with a guard block. Thus,

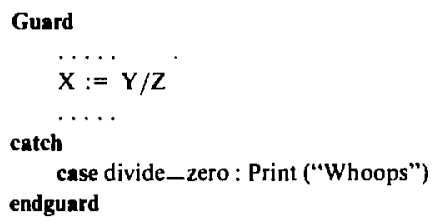

would catch any divide-by-zero exception in the code between the gu ird and catch phases, or in any nested routines invoked from within the code. When and if a named exception occurs. the first (deepest) dynamically nested catch case for the named exception is elaborated. The catch clause can specify various named exceptions as well as use a default tlause (i.e., all others).

Guard blocks may be used to contain exceptions in a large program or to catch an exception from a localized section. For instance, the Praxis input/output package uses exceptions for abnormal condition handling:

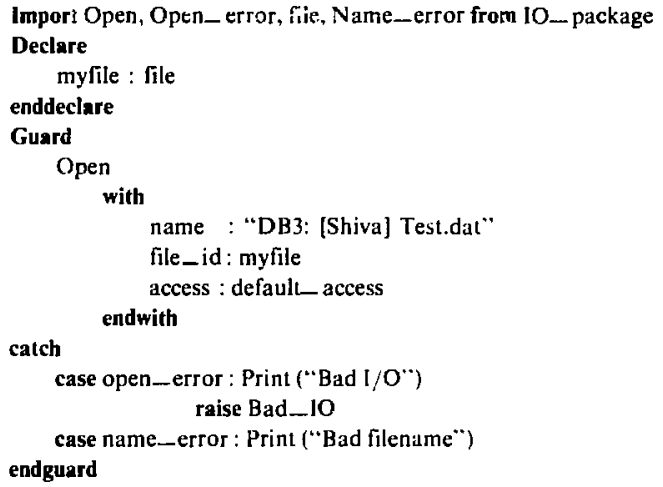

The Open procedure invocation is surrounded by a guard block: the procedure upon detecting an error will raise the exception open_err that is declared in the $1 O_{-}$- package. Control is transferred to the case clause in the catch block for the exception named. The clause is then elaborated. In the open-err case, a routine is invoked and then a user-defined exception is explicitly raised, and elaboration continues in a higher-level guard block. For the name-err exception, the case clause is elaborated and elaboration continues after the endguard. If no exceptions are raised within the open, then elaboration continues after the endguard. 
The Open example also introduced an alternate procedure invocation, using named formal parameters and the list (i.e., with-endwith) format. The named parameters allow the use of optional parameters and parameter specification in any order. The name on the left of the colon (:) is the name of the formal parameter, and the name on the right is the actual parameter of the invocation. The declaration of the Open procedure could be of the form

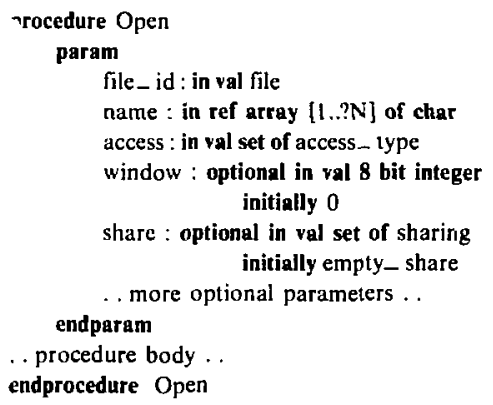

Only formal parameters declared as optional may be omitted in any actual invocation. Each formal parameter specified as optional must have a default value specified by the initially clause.

The Guard example introduced two procedures from the Input/Output package. The package is implemented as a series of procedures, functions. and abstract data types written in the language. Each implementation will have slightly different I/O packages, tailored to the particular operating environment. Under the PDP-11/RSX-11M and VAXiVMS operating systems, the I/O package supports the full RMS-I1 capabilities including indexed files. The standard, 1/O-related, encapsulated data types are

file record stream attribute

and some of the routines are

$\begin{array}{llll}\text { Create } & \text { Open } & \text { Close } & \text { Extend } \\ \text { Display } & \text { Erase } & \text { Connect } & \text { Disconnect } \\ \text { Find } & \text { Delete } & \text { Flush } & \text { Reiease } \\ \text { Get } & \text { Put } & \text { Rewind } & \text { Update }\end{array}$

In addition. a set of conversion routines for the predefined data types are supplied, which convert to/from ASCII text.

Other packages are supplied with implementations, or are supplied as interfaces to existing packages in other languages. Praxis routines can invoke other language subroutines and functions, or they may be called from other languages. For instance, a Fortran mathematics package would be defined as

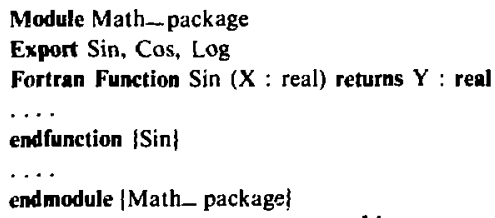


Other than the Furtran linkage. Praxis provides the linkages

Inline - Place routine code in place of invocation

Interrupt - PDP-II Interrupt service routine

Different compiler implementations could supply additional inkages.

An important feature that is necessary in the control environment is the ability to control the actual code generated for differing applications: for instance, the ability to generate code that would reside in ROM. This control is supplied by means of both predefined and user-defined compiler variables (comp_var), in conjunction with compiler directives. For inslance:

\%define Author, three_D

\%Set Author = "J R Greenberg"

$/ /$ string comp-var

\%Set Object_ROM

\% Set three $-\mathrm{D}=$ true

// predefined comp_.

$\because /$ user defined

\section{Declare}

span is $0 . .5$

Fif three _. D or : All_ three

data : array [span, span, span] of real

\%otherwise

data : array [span, span] of real

\%endif

enddeclare

Compiler vatiables can be either boolean or string yypes and are explicitly declared and assigned to by the \%Set compiler directive. The comp_-var Object-ROM specifies that the code generation should be such that the code and constant data can be burned into ROM. The \%if-\%otherwise-\%endif allows conditional compilation under control of a boolean comp_var expression. The referenced comp_var values can be set either within program text or upon compiler invocation.

Another feature that needs mentioning is the ability to generate specific instructions or nonstandard calling sequences. This is provided by the block-structured code statement shown below for a PDP-11 application:

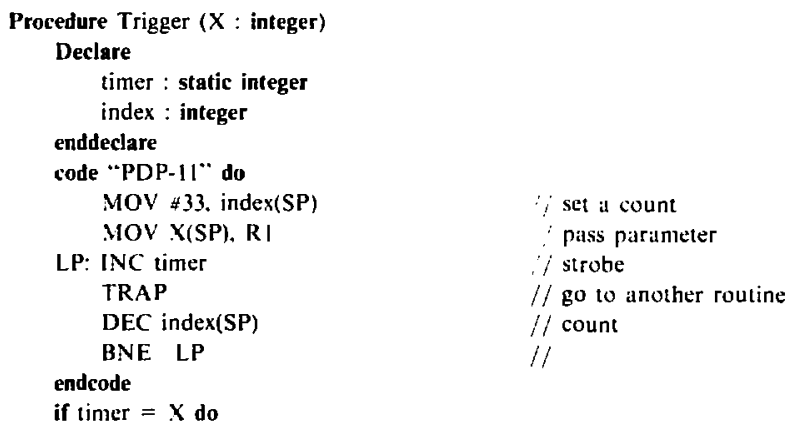




\section{endif \\ endprocedure $\{$ Trigger $\}$}

The concluding example outlines a simple task processur. using drays of procedure variables and the set data type:

\section{Declare}

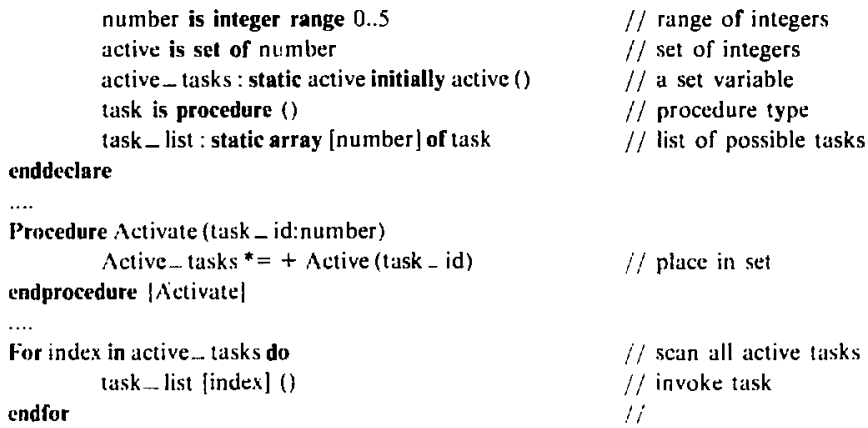

The set data tye in the declaration of active is used as an attribule associated with each rask. The set has six possible inembers denumerated by the values 0 through 5 . Stts wan be of any discrete type and can be arbitrarily large (i.e.. limited by memory size of machine). The active 11 after the initially clause and in the assignment statement is the set constant constructor. which allows items from the set to be included or removed. The For statement iterates through the set of active - tasks and will invoke any active task.

\section{Section 6}

\section{SUMMARY}

The preceding section, although introducing many fealures of the Praxis language. is by no means exhaustive. Some features have not been mentioned. and others have only been partially described. The full language is described in Praxis: Language Reference Wumul and the Programming in Praxis manual.

The Praxis language is specifically within the state of the art of language design, particularly designed for control and system implementation needs. Complex language features, such as generic procedures. overloading of operators, and parallel processes, have been intentionally left out. We felt that these concepts were either not understood enough to be incorporated at this time. or that they need not be part of the language.

In conclusion. Praxis is an extremely powerful. modern programming language that goes beyond Pascal and is available today. 


\section{ACKNOWLEDGMENTS}

The original language was designed by Arthur Evans. Jr., and C. Robert Morgan of BBN in 1977. Additional language design in 1979 by Evans and Morgan was augmented by James R. Greenwood (LLNL) and Michael C. Zarnstorff (University of Wisconsin). The final language design in 1980 was developed by the above individuals. with contributions from Earl Killian (BBN), Graeme Williams (BBN), and W. Nowicki (Stanford University).

The continued support of the management of the laser fusion program and the Nova laser project at LLNL, in particular J. L. Emmett. J. F. Holzrichter. R. O. Godwin. and W. W. Simmons, is gratefully acknowledged. The encouragement and support of $\mathrm{H}$. Ahistrom and $\mathrm{L}$. Coleman of the fusion experiments program at LLNL is also greatly appreciated.

The tremendous uffort by F. Holloway in developing the first application program in Praxis for the Nova control system is hereby acknowledged. His patience with early compiler releases, his persistence in developing the application acceptence test. and his constant enthusiasm were invaluable to the success of the project.

Additional thanks go to G. J. Suski, P. Rupert, and the controls development group at LLNL for their willingness to attempt the project and suffer through the preliminary versions of the product.

Also, the dedicated support and documentation efforts hy $W$. Nowicki was essential. In particular, his work on the Programming in Praxis manual came at a critical time.

The documentation and support role of $J$. Walker and R. Shapiro at BBN was extremely valuable. J. Walker created the Language Reference Manual in a short period of time from an everchanging definition. 


\section{BIBLIOGRAPHY}

Many languages are identified in the body of this report without specific references. Citations are as follows:

$\begin{array}{ll}\text { Ada } & \text { (Ichbiah-79A-79B) } \\ \text { ALGOL-60 } & \text { (Naur-63) } \\ \text { ALPHARD } & \text { (Wulf-76) } \\ \text { BCPL } & \text { (Richard-69), (BBN-74) } \\ \text { BLISS } & \text { (Wulf-7I) } \\ \text { CS-4 } & \text { (Intermetrics-75) } \\ \text { EUCLID } & \text { (Lampson-77) } \\ \text { FORTRAN } & \text { (FoRTRAN-76) } \\ \text { IMP } & \text { (Irons-70) } \\ \text { JOVIAL } & \text { (Shaw-63) } \\ \text { Mesa } & \text { (Mitchell-79) } \\ \text { Pascal } & \text { (Jensen-74) } \\ \text { PL/1 } & \text { (IBM) } \\ \text { Simula } & \text { (Dahl-70) }\end{array}$

(BBN-74)

BCPL Manual, Bolt, Beranek, and Newman, Inc.. Cambridge, Massachusetts (1954).

(Brinch-Hansen-72)

P. Brinch Hansen, "Structured Multiprogramming," Comm. $A C M$ 15, 7, 574-578 (1972).

(Brinch-Hansen-73)

P. Brinch-Hansen, Operoting Systems Principles, Prentice-Hall, Inc.. Englewood Cliffs, New Jersey (1973).

(Dahl-70)

O.-J. Dahl, B. Myhrhaug, and K. Nygaard, Common Base Language, Norwegian Computing Center, Publication S-22 (1970).

(DoD-77)

"Department of Defense Requirements for High-Order Computer Programming Language-Jronman," January 14, 1977.

(Evans-76)

A. Evans, Jr., and C. R. Morgan, Development of a Communications Oriented Language, Bolt. Beranek, and Newman. Inc., Cambridge, Massachusetts, Report No. 3261 (1976)

(Evans-77)

A. Evans. Jr., and C. R. Morgan, A Communications Oriented Language (COL): Language Design, Bolt, Beranek. and Newman, Inc. Cambridge, Massachusetts, Report No. 3534 (1977). 
(Fivins-79)

A. Evans. Jr., C. R. Morgan, E. S. Roberts, and E. M. Clarke. The Impact of lfultiprocessor Technology on High-Level Language Design. Bolt. Beranek. and Newman, Inc., Cambridge. Massachusetts, Report No. 4188 (1979).

(Fisher-76)

D. A. Fisher, "A Common Programming Language for the Department of Defense-Background and Technical Requirements," Institute for Defense Analysis, Paper P-1191. June 1976

(FORTRAN-76)

"Draft proposed ANS FORTRAN," $A C M$ Sigplan Notices 11. 3 (1976) (entire issue).

(1BM)

"PL/I Language Specification," IBM Corporation. ANSIl Standard for PL/I, Subset G. Form GY33-6003-2 (undated).

(Ichbiah-79A)

J. D. Ichbiah, J. Heiard, O. Roubine, J. Barnes, B. Krieg-Brueckner, and B. A. Wichmann, "Rationale for the Design of the A.da Programming Language." ACM Sigplan Notices 14, 6(1979).

(Ichbiah-79B)

J. D. Ichbiah, J. Heiard, O. Rour,ne, J. Barnes, B. Krieg-Brueckner, and B. A. Wichmann, "The Preliminary Ada Language Reference Manual," $A C M$ Sigplan Notices 14, 6 (1979).

(Intermetrics-75)

CS-4 Language Reference Mamual and Operating System Interface. Intermetrics, Inc., Cambridge. Massachusetts, Report IR-130-2 (1975).

(Irons-70)

E. T. Irons. "Experience with an Extensible Language," Comm. ACM 13, 1 (1970).

(Jensen-74)

K. Jensen and N. Wirth. PASCAL User Manual and Report (Second Edition). Springer-Verlag. Berlin (1974).

(Knuth-73)

D. E. Knuth, A Review of Structured Programming, Stanford University, Stanford, California, Computer Science Department. Report STAN-CS-73-371 (1973).

(Knuth-74)

D. E. Knuth. "Structured Programming with Goto Statements." Computing Surveys (December 1974).

(Lampson-77)

B. W. Lampson, J. J. Horning. R. L. London, J. G. Mitchel. and G. J. Popek. "Report on the Programming Language EUCLID." ACM Sigplan Notices 12. 2 (1977) (entire issue). 
(Mitchell-79)

J. G. Mitchell. W. Maybury. and R. Sweet, Mesa Language Mamual V.5. Xerox Corporation. Palo Alto, California. Report CSL-79-3 (1979).

(Morgan-77)

C. R. Morgan and A. Evans, Jr.. Communications Oriented Language ( $C O L)$ : Language Implementation. Bolt, Beranek, and Newman, Inc., Cambridge, Massachusetts, Report No. 3533 (1977).

(Naur-63)

"Revised Report on the Algorithmic Language ALGOL 60" (P. Naur, Ed.), Comm. AC.M 6. 1, 1-17 (1963).

(Richards-69)

M. Richi:rds, "BCPL $\rightarrow$ A Tool for Compiler Writing and Systems Progrannming," from Spring Joint Computer Conference (1969), pp. 557-566.

(Shaw-6.3)

C. J. Shaw. "A Specification of JOVIAL," Comm. ACM 6. 12, 721-736 (1963).

(Wirth-76)

v. Wirth. Algorithms + Data Structures $=$ Progrums. Prentice-Hall. Inc. Englewood C liffs. New Jersey (1976).

(Wuif-7])

IV. A. Wulf, D. B. Russell, and A. N. Haberman, "BLISS: A Language for System Programming," Comm. ACM 14. 12. 780-790 (1971).

(Wulf-76)

W. A. Wulf, R, L. London, and M. Shaw, sbstraction and Verification in ALPHARD: Introduction to Language and Methodology. Carnegie-Mellon University, Pittsburgh, Pennsylvania, Department of Computer Science (June 1976).

(Zahn-74)

C. T. Zahn, "A Control Structure for Natural Top Down Structured Programming." from Symposium on Programming Languages, Paris. France (1974). 


\section{Appendix \\ LANGUAGE SYNTAX}

\section{Backus-Naur Form (BNF)}

Here, we describe the context-free syntax of the language, using a variant of the Backus-Naur Form (BNF). In particular, we adhere to the following conventions in the BNF representation:

- Lower-case words, perhaps containing underscores, denote syntactic categories. such as:

$$
\begin{aligned}
& \text { function_list } \\
& \text { relation_operator } \\
& \text { linkage }
\end{aligned}
$$

- Boldface vords denote reserved words, for example.

$$
\begin{aligned}
& \text { select } \\
& \text { function } \\
& \text { or }
\end{aligned}
$$

- Square brackets enclose optional items. A quoted square bracket means that it is part of the syntax (i.e.. array subscripts and enumerations).

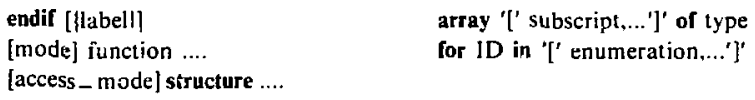

- Repeated items are represented by a delimiter followed by three dots. Thus. a list of identifiers could be designated by

identifier,...

where the comma is the repeat delimiter. Thus. the BNF form.

$$
\text { identifier_ list := identifier,... }
$$

means that the identifter list can contain one or more identifiers separated by commas. Another example is

$$
\text { statement } \_ \text {list }:=\text { statement } ; . .
$$

where the semicolon is the delimiter.

- The syntax rules describing structured constructs in the language are presented in a form that is visually similar to their usage in programs. For example, the select statement is specified in the BNF as 
select_statement := label: select expression from

[case case_literal, ... : sentence; ... ] ...

[default : sentence;....]

endselect \{lahcl\}

- Various syntactic items can be represented by the item prefixed by a qualifier corresponding to a category name. The prefix is intended to convey extra semantic information. For instance:

$$
\text { module_identifier module_ID function_identifier }
$$

are all equivalent to:

identifier

- Some abbreviations used in the syntax description are

$\begin{array}{ll}\text { ID } & \text { identifier } \\ \text { expr } & \text { expression } \\ \text { spec } & \text { specifier } \\ \text { c._constant } & \text { compile-time constant } \\ \text { I_ constant } & \text { link-time constant }\end{array}$

- The slash $(/)$ is used to delimit various cases of a BNF production. It can be read as "or."

Thus:

$$
\begin{aligned}
& \text { declaration }::=\text { procedure_declaration } \\
& \text { / function_declaration }
\end{aligned}
$$

is just shorthand for

$$
\begin{array}{ll}
\text { declaration } & :=\text { procedure_declaration } \\
\text { declaration } & :=\text { function_declaration }
\end{array}
$$

\section{Syntax Definition}

$$
\begin{aligned}
& \text { module declaration } \quad:=\text { [main] module module_- ID segment_list } \\
& \text { export ID.... [to module }- \text { ID .... ] .... } \\
& \text { sentence:... } \\
& \text { endmodule [\{module_ ID \}] } \\
& \text { module ID }::=\text { ID / module_. ID.ID } \\
& \text { sentence } \quad::=\text { statement / declaration / empty } \\
& \text { declaration } \quad::=\text { procedure }- \text { declaration } \\
& \text { / function-declaration } \\
& \text { / listed_declaration } \\
& \text { / import_declaration }
\end{aligned}
$$




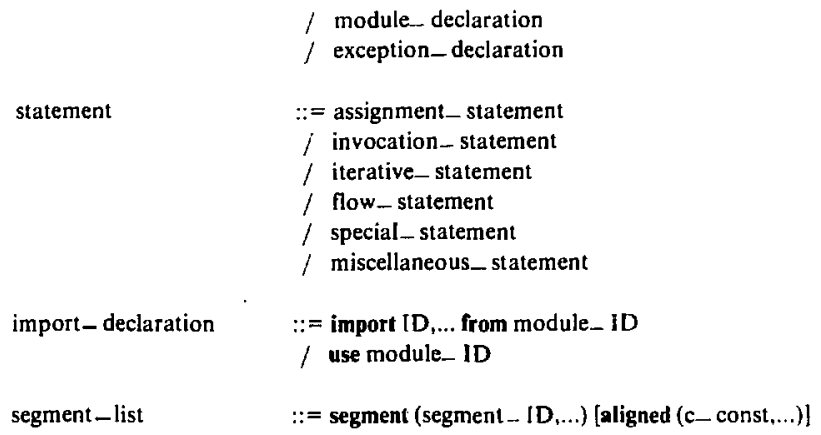

\section{Declarations}

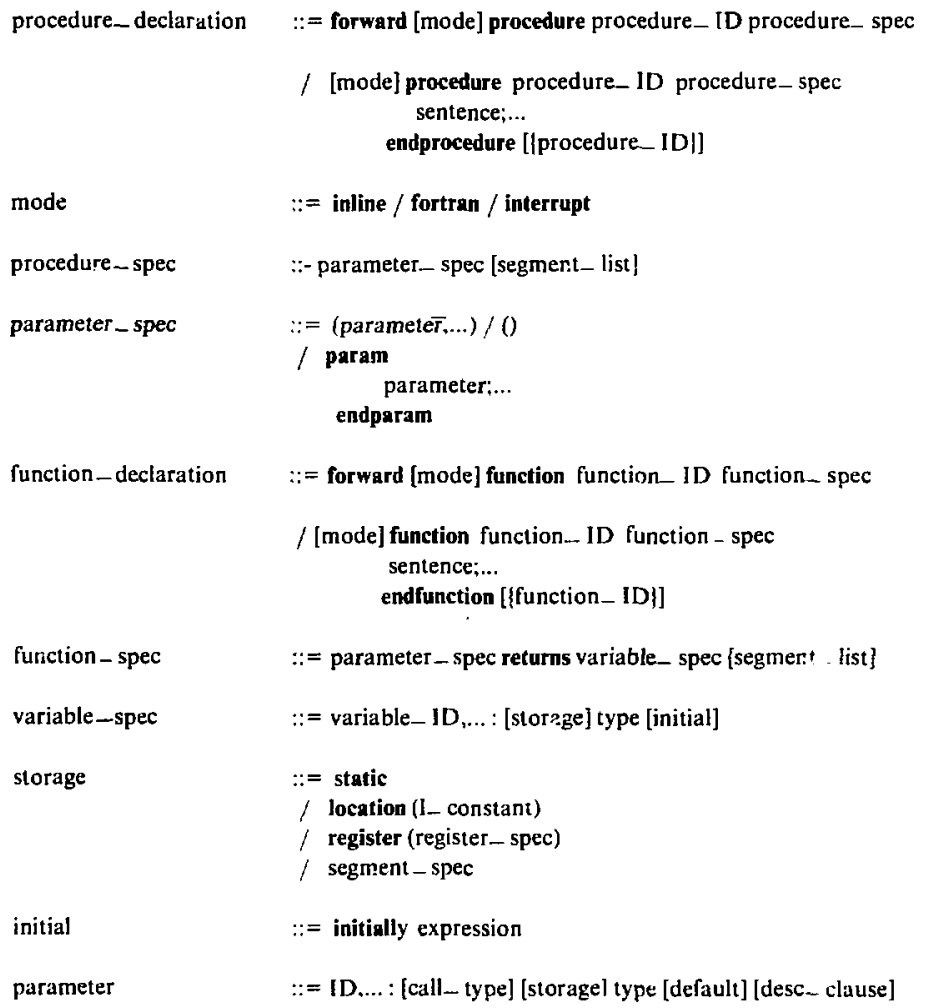




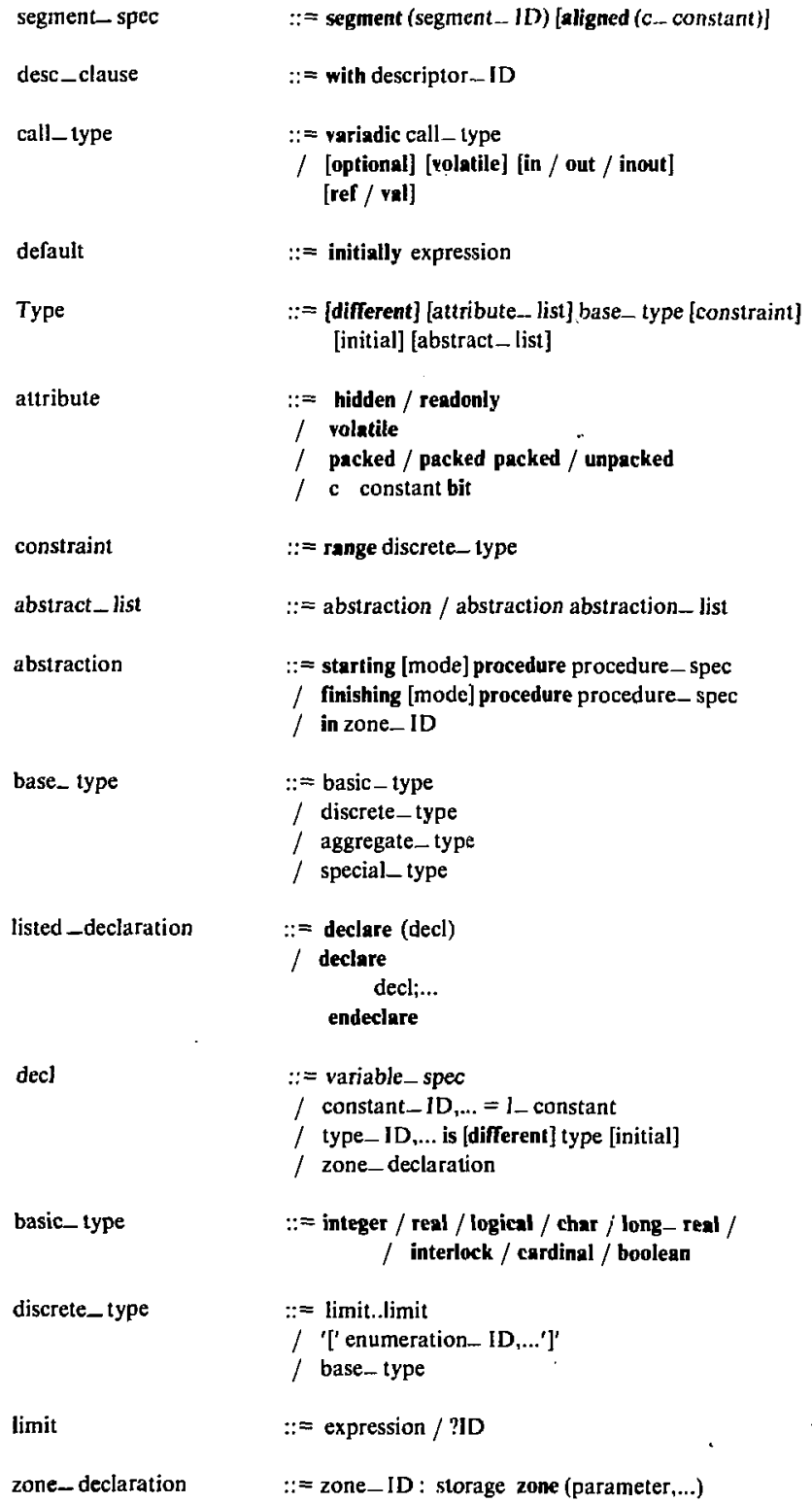




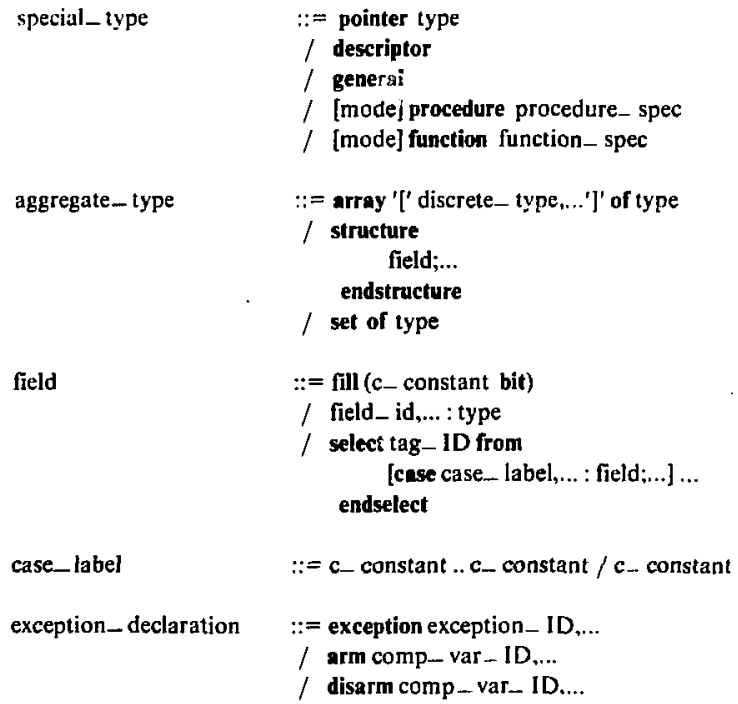

field

$::=$ fill $(c-$ constant bit)

$/$ field_ id,.... : type

/ select tag-ID from

[cese case_- label, ... : field;.... ... endselect

case_label

$::=c_{-}$constant .. c_- constant $/ c_{-}$constant

exception_declaration $\quad::=$ exception exception $\_$ID,...

/ arm comp_var_ID,...

/ disarm comp_var_. ID....

\section{Statements}

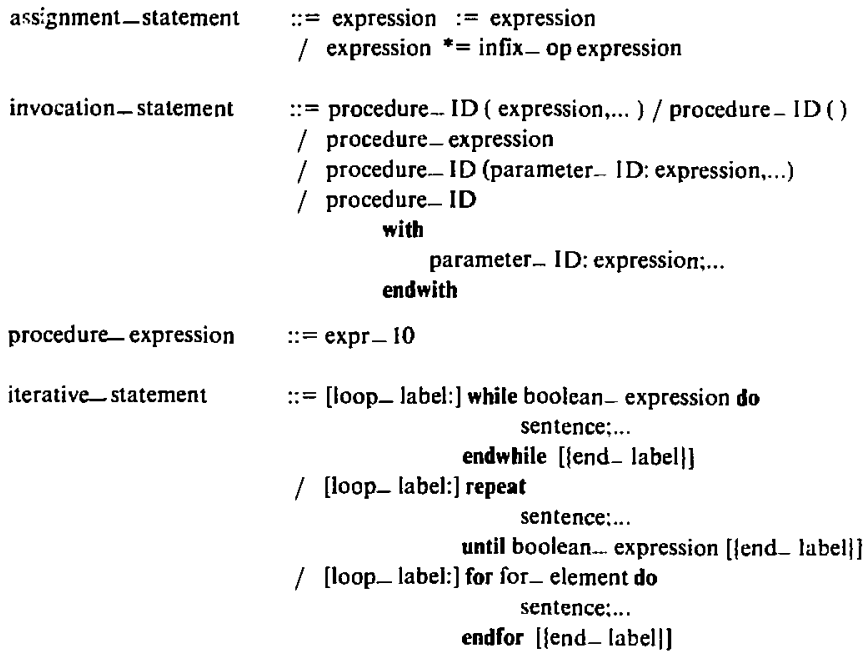




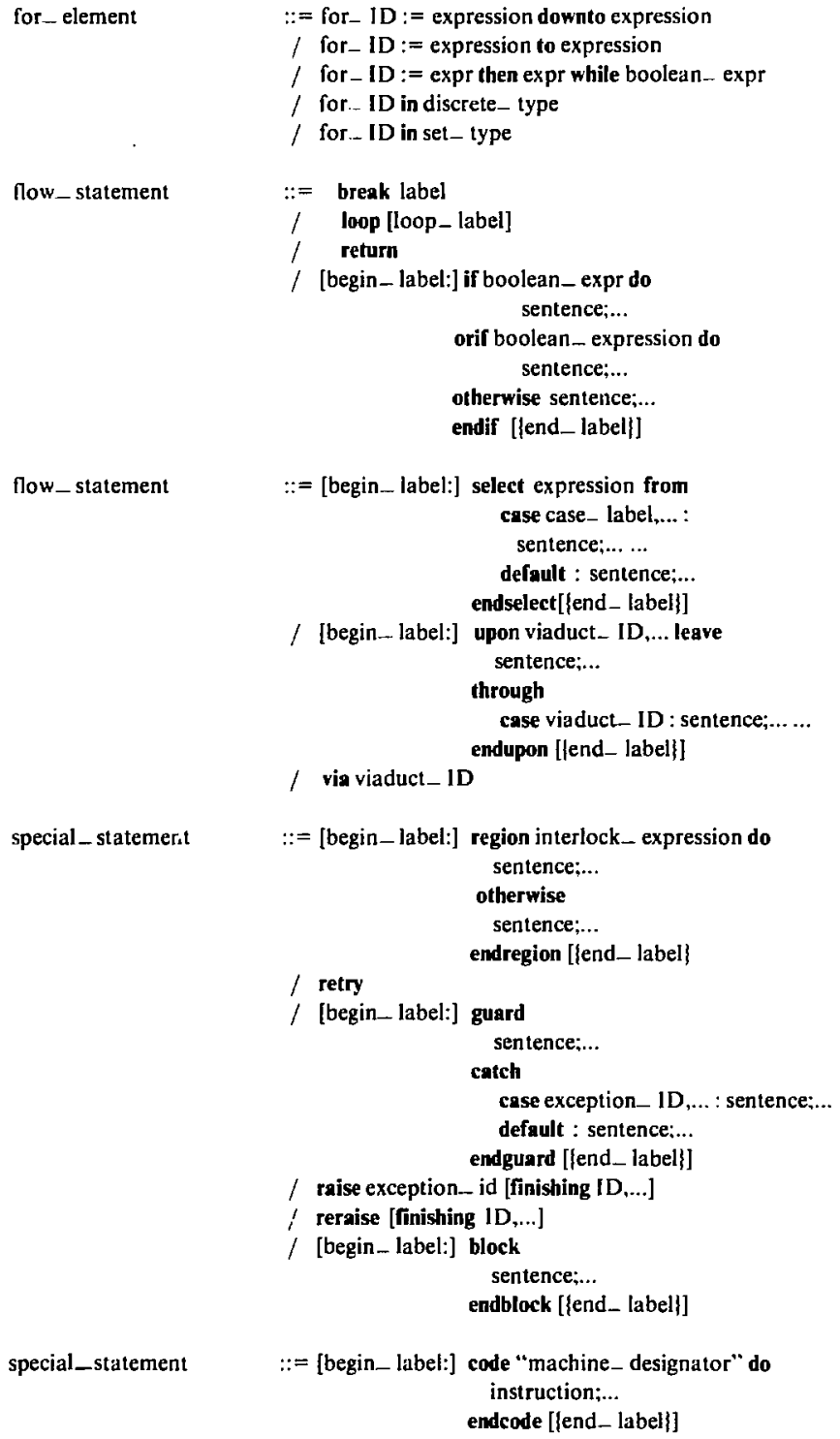




$$
\begin{array}{ll}
\text { instruction } & ::=\text { assembler_- instruction } \\
\text { misc. _tatement } & ::=\text { free (pointer_type.. expression....) } \\
& / \text { swap (expression. expression) } \\
& / \text { assert boolean_expression }
\end{array}
$$

\section{Expressions}

The numeric values on the "expr" identifiers below represent the operator precedence fevels.

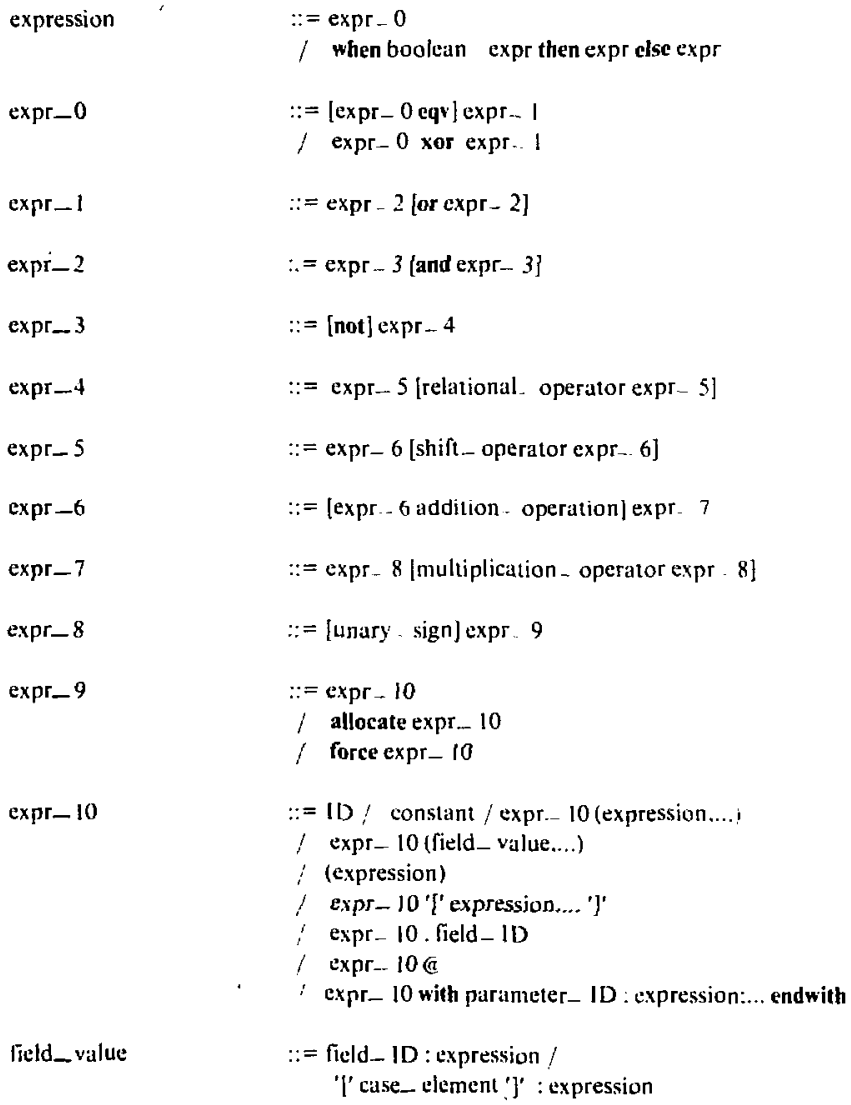




\section{Operators}

Infix - operitor

$::=$ eqv $/$ xor $/$ or $/$ and

/ relational_operator / shif__operator

/ addition-operator / multiplication - operator

relational operator

$::==|\langle>|<=|</\rangle=1\rangle$

shift_operator

$::=1$ sh / rsh

multiplication.- operator

$::=* / \% / \bmod$

\section{Predefined Functions}

\begin{tabular}{|c|c|}
\hline $\operatorname{mix}$ & - muximum \\
\hline $\min$ & - minimum \\
\hline suec & - successor \\
\hline pred & - predeccessor \\
\hline abs & - absolute value \\
\hline round & - real to integer rounded \\
\hline nloor & - largest intcger not greater than real \\
\hline ceiling & - smallest integer not less than real \\
\hline low & - lower limil of discrete type \\
\hline high & - upper limil or discrete type \\
\hline $\begin{array}{l}\text { size_- of } \\
\text { descriplor_of }\end{array}$ & $\begin{array}{l}\text { - size in bits of data object } \\
\text { - descriptor of a type }\end{array}$ \\
\hline
\end{tabular}

